\title{
A Multiple Attribute Group Decision Making Approach for Solving Problems with the Assessment of Preference Relations
}

\author{
Taho Yang, ${ }^{1}$ Yiyo Kuo, ${ }^{2}$ David Parker, ${ }^{3}$ and Kuan Hung Chen ${ }^{1}$ \\ ${ }^{1}$ Institute of Manufacturing Information and Systems, National Cheng Kung University, Tainan 70101, Taiwan \\ ${ }^{2}$ Department of Industrial Engineering and Management, Ming Chi University of Technology, New Taipei City 24301, Taiwan \\ ${ }^{3}$ The University of Queensland Business School, Brisbane, QLD 4072, Australia \\ Correspondence should be addressed to Yiyo Kuo; yiyo@mail.mcut.edu.tw
}

Received 19 June 2014; Revised 21 October 2014; Accepted 23 October 2014

Academic Editor: Mu-Chen Chen

Copyright (C) 2015 Taho Yang et al. This is an open access article distributed under the Creative Commons Attribution License, which permits unrestricted use, distribution, and reproduction in any medium, provided the original work is properly cited.

\begin{abstract}
A number of theoretical approaches to preference relations are used for multiple attribute decision making (MADM) problems, and fuzzy preference relations is one of them. When more than one person is interested in the same MADM problem, it then becomes a multiple attribute group decision making (MAGDM) problem. For both MADM and MAGDM problems, consistency among the preference relations is very important to the result of the final decision. The research reported in this paper is based on a procedure that uses a fuzzy preference relations matrix which satisfies additive consistency. This matrix is used to solve multiple attribute group decision making problems. In group decision problems, the assessment provided by different experts may diverge considerably. Therefore, the proposed procedure also takes a heterogeneous group of experts into consideration. Moreover, the methods used to construct the decision matrix and determine the attribution of weight are both introduced. Finally a numerical example is used to test the proposed approach; and the results illustrate that the method is simple, effective, and practical.
\end{abstract}

\section{Introduction}

There are many situations in daily life and in the workplace which pose a decision problem. Some of them involve picking the optimum solution from among multiple available alternatives. Therefore in many domain problems multiple attribute decision making methods, such as simple additive weighting (SAW), the technique for order preference by similarity to ideal solution (TOPSIS), analytical hierarchy process (AHP), data envelopment analysis (DEA), or grey relational analysis (GRA) [1-5], are usually adopted, for example, layout design [6-8], supply chain design [9], push/pull junction point selection [10], pacemaker location determination [11], work in process level determination [12], and so on.

If more than one person is involved in the decision, the decision problem becomes a group decision problem. Many organizations have moved from a single decision maker or expert to a group of experts (e.g., Delphi) to accomplish this task successfully $[13,14]$. Note that an "expert" represents an authorized person or an expert who should be involved in this decision making process. However, no single alternative works best for all performance attributes, and the assessment of each alternative given by different decision makers may diverge considerably. As a consequence, multiple attribute group decision making (MAGDM) is more difficult than cases where a single decision maker decides using a multiple attribute decision making method.

MAGDM is one of the most common activities in modern society, which involves selecting the optimal one from a finite set of alternatives with respect to a collection of the predefined criteria by a group of experts with a high collective knowledge level on these particular criteria [15]. When a group of experts wants to choose a solution from among several alternatives, preference relations is one type of assessment that experts could provide. Preference relations are comparisons between two alternatives for a particular attribute. A higher preference relation means that there is a higher degree of preference for one alternative over another. 
However, different experts may use different assessment types to express the preference relation. It is possible that in group decision making different experts express their preference in different formats [16-21].

In addition, after experts have provided their assessment of the preference relation, the appropriateness of the comparison from each expert must be tested. Consistency is one of the important properties for verifying the appropriateness of choices [22]. If the comparison from an expert is not logically consistent for a specific attribute, it means that at least one preference relation provided by the expert is defective. Therefore, the lack of consistency in decision making can lead to inconsistent conclusions.

Quite apart from the type of assessment, there can be considerable variation between experts as to their evaluation of the degree of the preference relation. In general, it would be possible to aggregate the preferences of experts by taking the weight assigned by every expert into consideration. However, heterogeneity among experts should also be considered [23]. For example, if the expert who assigns the greatest weight to a preference relation also makes choices that are not appropriate and quite different from the evaluations of the other experts who assign lower weights, then the group decision procedure can be distorted and imperfect.

Moreover, the determination of attribute weight is also an important issue [24]. In some decision cases, some attributes are considered to be more important in the experts' professional judgment. However, for these important attributes, the preference relation provided by experts may be quite similar for all alternatives. Even for the attribute with the highest weight, the degree of influence on the final decision would be very small in this case. In this way this kind of attribute can become unimportant to the final decision [25].

Therefore, during the multiple attribute group decision process, 5 aspects should be noted:

(i) considering different assessment types simultaneously;

(ii) insuring the preference relations provided by experts are consistent;

(iii) taking heterogeneous experts into consideration;

(iv) deciding the weight of each attribute;

(v) ranking all alternatives.

Group decision making has been addressed in the literature. In recent years, Ölçer and Odabaşi [23] proposed a fuzzy multiple attribute decision making method to deal with the problem of ranking and selecting alternatives. Experts provide their opinion in the form of a trapezoidal fuzzy number. These trapezoidal fuzzy numbers are then aggregated and defuzzified into a MADM. Finally, TOPSIS is used to rank and select alternatives. In the method, experts can provide their opinion only by trapezoidal fuzzy number.

Boran et al. [26] proposed a TOPSIS method combined with intuitionistic fuzzy set to select appropriate supplier in group decision making environment. Intuitionistic fuzzy weighted averaging (IFWA) operator is utilized to aggregate individual opinions of decision makers for rating the importance of criteria and alternatives. Cabrerizo et al. [27] presented a consensus model for group decision making problems with unbalanced fuzzy linguistic information. This consensus model is based on both a fuzzy linguistic methodology to deal with unbalanced linguistic term sets and two consensus criteria-consensus degrees and proximity measures. Chuu [28] builds a group decision making model using fuzzy multiple attributes analysis to evaluate the suitability of manufacturing technology. The proposed approach involved developing a fusion method of fuzzy information, which was assessed using both linguistic and numerical scales.

$\mathrm{Lu}$ et al. [29] developed a software tool for supporting multicriteria group decision making. When using the software, after inputting all criteria and their corresponding weights, and the weighting for all the experts, all the experts can assess every alternative against each attribute. Then the ranking of all alternatives can be generated. In the software only one assessment type is allowed and there is no function that can be used to ensure that the preference relations provided by experts are consistent. Zhang and Chu [30] proposed a group decision making approach incorporating two optimization models to aggregate these multiformat and multigranularity linguistic judgments. Fuzzy set theory is utilized to address the uncertainty in the decision making process.

Cabrerizo et al. [14] proposed a consensus model to deal with group decision making problems, in which experts use incomplete unbalanced fuzzy linguistic preference relations to provide their preference. However, the model requires that preference relations should be assessed in the same way, and no allowance is made for heterogeneous experts. Cebi and Kahraman [31] proposed a methodology for group decision support. The methodology consists of eight steps which are (1) definition of potential decision criteria, possible alternatives, and experts, (2) determining the weighting of experts, (3) identifying the importance of criteria, (4) assigning alternatives, (5) aggregating experts' preferences, (6) identifying functional requirements, (7) calculating information contents, and (8) calculating weighted total information contents and selecting the best alternative. The methodology does not include a check on the consistency of preference relations provided by the experts.

The novelty of the present study is that it proposes a multiple attribute group decision making methodology in which all of the five issues mentioned above are addressed. A review of the literature related to this research suggests that no previous research has addressed all of the issues simultaneously. For managers who are not experts in fuzzy theory, group decision making, MADM, and so on, this research can provide a complete guideline for solving their multiple attribute group decision making problem.

The remainder of this paper is organized as follows. In Section 2 all the issues set out above are discussed and appropriate methodologies for dealing with them are proposed. Then an overall approach is proposed in Section 3. The proposed model is tested and examined with a numerical example in Section 4. Finally Section 5 contains the discussion and conclusions. 


\section{Multiple Attribute Group Decision Making Methodology}

2.1. Assessment and Transformation of Preference Relations. There are two types of preference relations that are widely used. One is fuzzy preference relations, in which $r_{i j}$ denotes the preference degree or intensity of the alternative $i$ over $j$ [32-35]. If $r_{i j}=0.5$, it means that alternatives $i$ and $j$ are indifferent; if $r_{i j}=1$, it means that alternative $i$ is absolutely preferred to $j$, and if $r_{i j}>0.5$, it means that alternative $i$ is preferred to $j . r_{i j}$ is reciprocally additive; that is, $r_{i j}+r_{j i}=1$ and $r_{i i}=0.5[35,36]$

The other widely used type of preference relations is multiplicative preference relations, in which $a_{i j}$ indicates a ratio of preference intensity for alternative $i$ to that of alternative $j$; that is, it is interpreted as meaning that alternative $i$ is $a_{i j}$ times as good as alternative $j$ [17]. Saaty [3] suggested measuring $a_{i j}$ on an integer scale ranging from 1 to 9 . If $a_{i j}=1$, it means that alternatives $i$ and $j$ are indifferent; if $a_{i j}=9$, it means that alternative $i$ is absolutely preferred to $j$, and if $8 \geq r_{i j} \geq 2$, it means that alternative $i$ is preferred to $j$. In addition, $a_{i j} \times a_{j i}=1$, and $a_{i j}=a_{i k} \times a_{k j}$.

For these two preference types, Chiclana et al. [17] proposed an equation to transform the multiplicative preference relation into the fuzzy preference relation, as shown by

$$
r_{i j}=0.5\left(1+\log _{9} a_{i j}\right) .
$$

However, for both preference types, it is possible that some experts would not wish to provide their preference relation in the form of a precise value. In the fuzzy preference relations, experts can use the following classifications:

(i) a precise value, for example, " 0.7 ";

(ii) a range, for example, $(0.3,0.7)$; the value is likely to fall between 0.3 and 0.7 ;

(iii) a fuzzy number with triangular membership function, for example, $(0.4,0.6,0.8)$; the value is between 0.4 and 0.8 and is most probably 0.6 ;

(iv) a fuzzy number with trapezoidal membership function, for example, $(0.3,0.5,0.6,0.8)$; the value is between 0.3 and 0.8 , most probably between 0.5 and 0.6 .

In this paper, the four classifications set out above are unified by transferring them into trapezoidal membership functions. Thus, 0.7 becomes $(0.7,0.7,0.7,0.7),(0.3,0.7)$ becomes $(0.3,0.3,0.7,0.7)$, and $(0.4,0.6,0.8)$ then becomes $(0.4,0.6,0.6,0.8)$. If experts provide their assessment in the format of multiplicative preference relations, it will be transformed into a trapezoidal membership function first, and then using (1) it will be further transformed into the format of fuzzy preference relations. For example, $(3,4,5$, 6) can be transferred into $(0.75,0.82,0.87,0.91)$ by using (1). Therefore, this paper will mention only fuzzy preference relations in what follows.

2.2. The Generation of Consistent Preference Relations. The property of consistency has been widely used to establish a verification procedure for preference relations, and it is very important for designing good decision making models [22]. In the analytical hierarchy process, for example, in order to avoid potential comparative inconsistency between pairs of categories, a consistency ratio (CR), an index for consistency, has been calculated to assure the appropriateness of the comparisons [3]. If the CR is small enough, there is no evidence of inconsistency. However, if the CR is too high, then the experts should adjust their assessments again and again until the CR decreases to a reasonable value. For fuzzy preference relations, Herrera-Viedma et al. [22] designed a method for constructing consistent preference relations which satisfy additive consistency. Using this method, all experts need only to provide preference relations between alternatives $i$ and $i+1, r_{i(i+1)}$, and the remaining preference relations can be calculated using (2) if $i>j$ and (3) if $i<j$ :

$$
\begin{aligned}
& r_{i j}=\frac{i-j+1}{2}-r_{j(j+1)}-r_{(j+1)(j+2)}-\cdots-r_{(i-1) i}, \quad \forall i>j, \\
& r_{i j}=1-r_{j i}, \quad \forall i<j .
\end{aligned}
$$

To illustrate the generation of preferential relations, we provide an empirical example of four alternatives as follows. First, the expert provides the three preference relations as $r_{12}=0.3, r_{23}=0.6$, and $r_{34}=0.8$.

According to (2),

$$
\begin{aligned}
& r_{21}=1-0.3=0.7, \\
& r_{31}=1.5-0.3-0.6=0.6, \\
& r_{41}=2-0.3-0.6-0.8=0.3, \\
& r_{32}=1-0.6=0.4, \\
& r_{42}=1.5-0.6-0.8=0.1, \\
& r_{43}=1-0.8=0.2 .
\end{aligned}
$$

According to (3),

$$
\begin{aligned}
& r_{13}=1-0.6=0.4 \\
& r_{14}=1-0.3=0.7 \\
& r_{24}=1-0.1=0.9 .
\end{aligned}
$$

Therefore, the preference relations matrix, $\mathrm{PR}$, is

$$
\mathrm{PR}=\left[\begin{array}{llll}
0.5 & 0.3 & 0.4 & 0.7 \\
0.7 & 0.5 & 0.6 & 0.9 \\
0.6 & 0.4 & 0.5 & 0.8 \\
0.3 & 0.1 & 0.2 & 0.5
\end{array}\right]
$$

In general, experts are asked to evaluate all pairs of alternatives and then construct a preference matrix with full information. However, it is difficult to obtain a consistent preference matrix in practice, especially when measuring preferences on a set with a large number of alternatives [22]. 
2.3. Assessment Aggregation for a Heterogeneous Group of Experts. For each comparison between a pair of alternatives, the preference relations provided by different experts would vary. Hsu and Chen [37] proposed an approach to aggregate fuzzy opinions for a heterogeneous group of experts. Then, Chen [38] modified the approach and Ölçer and Odabaşi [23] present it as the following six-step procedure.

(1) Calculate the Degree of Agreement between Each Pair of Experts. For a comparison between two alternatives, let there be $E$ experts in the decision group, $\left(a_{1}, a_{2}, a_{3}, a_{4}\right)$ and $\left(b_{1}, b_{2}, b_{3}, b_{4}\right)$ are the preference relations provided by experts $a$ and $b, 1 \leq a \leq E, 1 \leq b \leq E$, and $a \neq b$. The similarity between these two trapezoidal fuzzy numbers, $S_{a b}$, can be measured by

$$
S_{a b}=1-\frac{\left|a_{1}-b_{1}\right|+\left|a_{2}-b_{2}\right|+\left|a_{3}-b_{3}\right|+\left|a_{4}-b_{4}\right|}{4} .
$$

(2) Construct the Agreement Matrix. After all the agreement degrees between experts are measured, the agreement matrix (AM) can be constructed as follows:

$$
\mathrm{AM}=\left[\begin{array}{cccc}
1 & S_{12} & \cdots & S_{1 E} \\
S_{21} & 1 & \cdots & S_{2 E} \\
\vdots & \vdots & S_{a b} & \vdots \\
S_{E 1} & S_{E 2} & \cdots & 1
\end{array}\right]
$$

in which $S_{a b}=S_{b a}$, and if $a=b$, then $S_{a b}=1$.

(3) Calculate the Average Degree of Agreement for Each Expert. The average degree of agreement for expert $a\left(\mathrm{AA}_{a}\right)$ can be calculated by

$$
\mathrm{AA}_{a}=\frac{1}{E-1} \sum_{b=1, a \neq b}^{E} S_{a b}, \quad \forall a
$$

(4) Calculate the Relative Degree of Agreement for Each Expert. After calculating the average degree of agreement for all experts, the relative degree of agreement for expert $a\left(\mathrm{RA}_{a}\right)$ can be calculated by

$$
\mathrm{RA}_{a}=\frac{\mathrm{AA}_{a}}{\sum_{a=1}^{E} \mathrm{AA}_{a}}, \quad \forall a
$$

(5) Calculate the Coefficient for the Degree of Consensus for Each Expert. Let $\mathrm{ew}_{a}$ be the weight of expert $a$, and $\sum_{a=1}^{E} \mathrm{ew}_{a}=1$. The coefficient of the degree of consensus for expert $a\left(\mathrm{CC}_{a}\right)$ can be calculated by

$$
\mathrm{CC}_{a}=\beta \cdot \mathrm{ew}_{a}+(1-\beta) \cdot \mathrm{RA}_{a}, \quad \forall a,
$$

in which $\beta$ is a relaxation factor of the proposed method and $0 \leq \beta \leq 1$. It represents the importance of $\mathrm{ew}_{a}$ over $\mathrm{RA}_{a}$.
When $\beta=0$, it means that the group of experts is considered to be homogeneous.

(6) Calculate the Aggregation Result. Finally, the aggregation result of the comparison between two alternatives $i$ and $j$ is $\tilde{r}_{i j}$, where

$$
\begin{aligned}
\tilde{r}_{i j}= & \mathrm{CC}_{1} \otimes \widetilde{r}_{i j}(1) \oplus \mathrm{CC}_{2} \otimes \tilde{r}_{i j}(2) \oplus \cdots \oplus \mathrm{CC}_{a} \\
& \otimes \widetilde{r}_{i j}(a) \oplus \cdots \oplus \mathrm{CC}_{E} \otimes \widetilde{r}_{i j}(E) .
\end{aligned}
$$

In (12), $\widetilde{r}_{i j}(a)$ is the preference relation between alternatives $i$ and $j$ provided by expert $a$, and $\widetilde{r}_{i j}=\left(r_{i j}^{1}, r_{i j}^{2}, r_{i j}^{3}, r_{i j}^{4}\right)$. Moreover $\otimes$ and $\oplus$ are the fuzzy multiplication operator and the fuzzy addition operator, respectively.

Let there be $N$ alternatives. Since each expert only provides preference relations between alternatives $i$ and $i+$ 1 , the aggregation process for a heterogeneous group of experts must be executed $N-1$ times in order to generate $N-1$ aggregated trapezoidal fuzzy numbers. These $N-$ 1 trapezoidal fuzzy numbers can then be converted into a precise value by the use of

$$
r_{i j}=\frac{r_{i j}^{1}+2\left(r_{i j}^{2}+r_{i j}^{3}\right)+r_{i j}^{4}}{6} .
$$

After the aggregation procedure, using (2) and (3), an aggregated preference relations matrix for attribute $k$ is constructed as follows:

$$
\mathrm{PR}_{k}=\left[\begin{array}{cccc}
1 & r_{12} & \cdots & r_{1 N} \\
r_{12} & 1 & \cdots & r_{2 N} \\
\vdots & \vdots & 1 & \vdots \\
r_{N 1} & r_{N 2} & \cdots & 1
\end{array}\right]
$$

2.4. Attribute Weight Determination. In a preference relations matrix of attribute $k, r_{i j}$ indicates the degree of preference of alternative $i$ over $j$ when attribute $k$ was considered. Therefore, $\sum_{j=1, j \neq i}^{N} r_{i j}$ indicates total degree of preference of alternative $i$ over the other $N-1$ alternatives. In the same way, $\sum_{j=1, j \neq i}^{N} r_{j i}$ indicates the total degree of preference of the other $N-1$ alternatives over alternative $i$. Fodor and Roubens [39] proposed (15) to define $\delta_{i k}$, the net degree of preference of alternative $i$ over the other $N-1$ alternatives by attribute $k$, and the bigger $\delta_{i k}$ is, the better alternative $i$ by attribute $k$ is:

$$
\delta_{i k}=\sum_{j=1, j \neq i}^{N} r_{i j}-\sum_{j=1, j \neq i}^{N} r_{j i}, \quad \forall i, k .
$$

Thus, the problem is reduced to a multiple attribute decision making problem:

$$
\mathrm{DM}=\left[\begin{array}{cccc}
\delta_{11} & \delta_{12} & \cdots & \delta_{1 M} \\
\delta_{21} & \delta_{22} & \cdots & \delta_{2 M} \\
\vdots & \vdots & \vdots & \vdots \\
\delta_{N 1} & \delta_{N 2} & \cdots & \delta_{N M}
\end{array}\right] .
$$


For the decision matrix constructed in Section 2.4, Wang and Fan [25] proposed two approaches, absolute deviation maximization (ADM) and standard deviation maximization (SDM), to determine the weight of all attributes. For a certain attribute, if the difference of the net degree of preference among all alternatives shows a wide variation, this means this attribute is quite important. ADM and SDM used absolute deviation (AD) and standard deviation (SD) to measure the degree of variation. An attribute with a bigger value of $\mathrm{AD}$ and SD will be a more important attribute.

When ADM was adopted, the weight of attribute $k, \mathrm{aw}_{k}$, was calculated by using (17), while if SDM was adopted, (18) was used for calculating the weight of attribute $k$ :

$$
\begin{aligned}
\mathrm{aw}_{k} & =\frac{\left(\sum_{i=1}^{N} \sum_{j=1}^{N}\left|\delta_{i k}-\delta_{j k}\right|\right)^{1 /(p-1)}}{\sum_{l=1}^{M}\left(\sum_{i=1}^{N} \sum_{j=1}^{N}\left|\delta_{i l}-\delta_{j l}\right|\right)^{1 /(p-1)}}, \quad \forall k ; p>1, \\
\mathrm{aw}_{k} & =\frac{\left(\sum_{i=1}^{N} \delta_{i k}^{2}\right)^{1 / 2(p-1)}}{\sum_{l=1}^{M}\left(\sum_{i=1}^{N} \delta_{i l}^{2}\right)^{1 / 2(p-1)}}, \quad \forall k ; p>1,
\end{aligned}
$$

where $p$ is the parameter of these two functions for calculating weights. Setting the variable to different values will lead to different weights and when $p=\infty$, all weights will be equal. Therefore, in order to reflect the differences among the attribute weights, Wang and Fang [25] suggested preferring a small value for parameter $p$. Further details of the demonstration of the use of ADM and SDM can be found in the paper by Wang and Fan [25].

2.5. Alternative Ranking. Once the weights of all attributes are determined by (17) or (18), the multiple attribute decision making problem constructed by (16) can be solved by the application of a multiple attribute decision making method, such as SAW, TOPSIS, ELECTRE, or GRA $[1,2,5]$. According to Kuo et al. [40], different MADM methods would lead to different results, but similar ranking of alternatives. In this research, SAW was selected for the MADM problem. Since the weight calculated by (17) and (18) has been normalized, and $\sum_{k=1}^{M} \mathrm{aw}_{k}=1$, the score of alternatives $i, C_{i}$, can be calculated directly by

$$
C_{i}=\sum_{k=1}^{M} \mathrm{aw}_{k} \delta_{i k}, \quad i=1,2, \ldots, N .
$$

The bigger the $C_{i}$ is, the better the alternative $i$ is. After the scores of all alternatives have been calculated, the alternatives can be ranked by $C_{i}$.

\section{The Proposed Approach}

Following from the consideration of issues which were set out in the Introduction and further developed in Section 2, this research proposes a 5-step procedure for multiple attribute group decision making problems as shown in Figure 1.

In Step 1, experts provide their preference relations for all attributes using their preferred format of expression. In

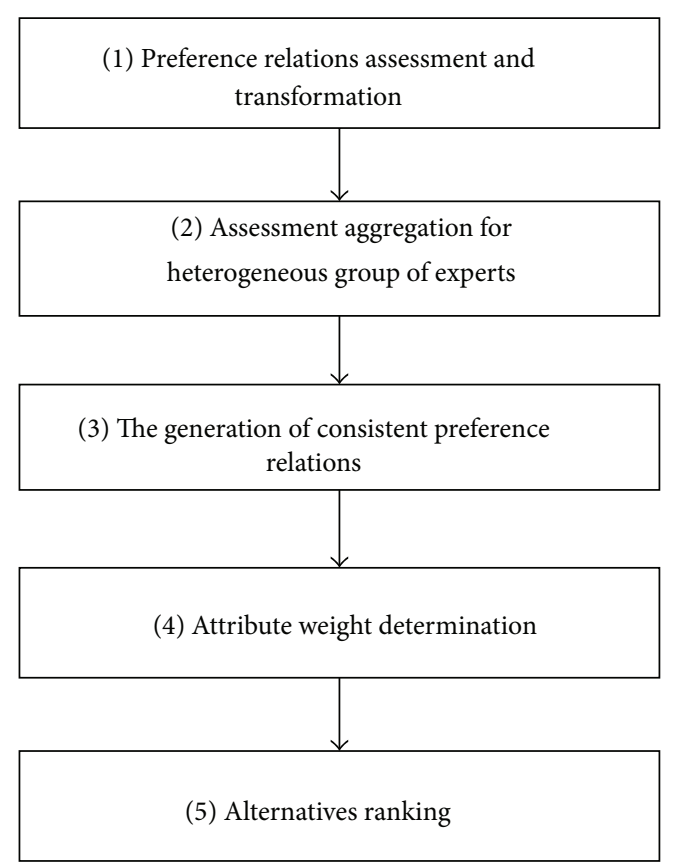

FIgURE 1: The proposed MAGDM procedure.

order to ensure the additive consistency of these preference relations, only the preference relations between alternatives $i$ and $i+1$ are assessed. Then these preference relations provided by the experts are transformed into trapezoidal membership functions. If the preference relations are multiplicative preference relations, (1) is used to transform them into fuzzy preference relations.

In Step 2, in order to take the heterogeneity of the experts into consideration, the trapezoidal membership function of fuzzy preference relations for all experts is aggregated by a sixstep procedure given by Ölçer and Odabaşi [23]. Then (2) and (3) are used to calculate the remaining preference relations which had not been provided by the experts, and these are then used to construct preference relation matrixes which are additively consistent in Step 3.

In Step 4, these preference relation matrixes are transformed into a traditional multiple attribute decision matrix and used to determine the weight of all attributes using (17) and (18). Finally, all the scores of alternatives can be calculated using (19) and the alternatives can be ranked in Step 5.

\section{Numerical Example}

The proposed MAGDM methodology allows two types of preference relations, fuzzy reference relations and multiplicative preference relations, which are explained in Section 2.1. The former ones are transformed to numerical number through fuzzy membership functions and the latter ones directly use numerical numbers. They are then aggregated through the proposed aggregation and ranking procedure as discussed in Sections 2.2 to 2.5. Due to both the transformation and aggregation procedures, the resulting numbers are real numbers. 
In this section, we provide a numerical example to illustrate the implementation of the proposed methodology. Consider four alternatives, three experts, and two attribute MAGDM problems as follows.

Step 1 (preference relations assessment and transformation). The preference relations assessments of Attribute 1 provided by these three experts were given as follows. in which $R_{a k}$ is the assessment of attribute $k$ provided by expert $a$ :

$$
\begin{aligned}
R_{11} & =\left[\begin{array}{cccc}
- & \text { Low } & - & - \\
- & - & \text { Low } & - \\
- & - & - & \text { Medium } \\
- & - & - & -
\end{array}\right], \\
R_{21} & =\left[\begin{array}{cccc}
- & \text { More low } & - & - \\
- & - & \text { Medium } & - \\
- & - & - & \text { Medium } \\
- & - & - & -
\end{array}\right],
\end{aligned}
$$

$$
R_{31}=\left[\begin{array}{llll}
- & \frac{1}{3} & - & - \\
- & - & \frac{1}{4} & - \\
- & - & - & 1 \\
- & - & - & -
\end{array}\right] .
$$

In this example, Experts 1 and 2 preferred to provide assessment by fuzzy preference relations, and Expert 3 preferred to provide assessment by multiplicative preference relations. However, Expert 1 used the membership function as shown in Figure 2, Expert 2 used the membership function as shown in Figure 3, and Expert 3 used precise values for providing his/her preference relations. All assessments are then transformed into the type of trapezoidal membership function as shown below:

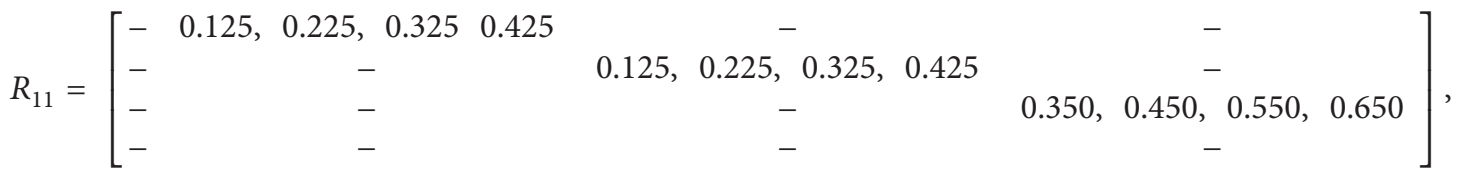

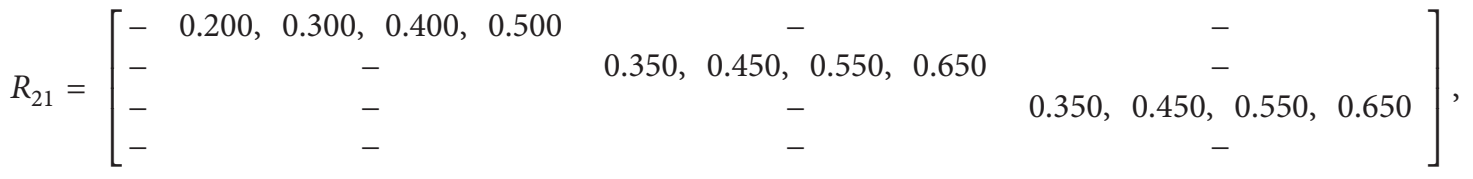

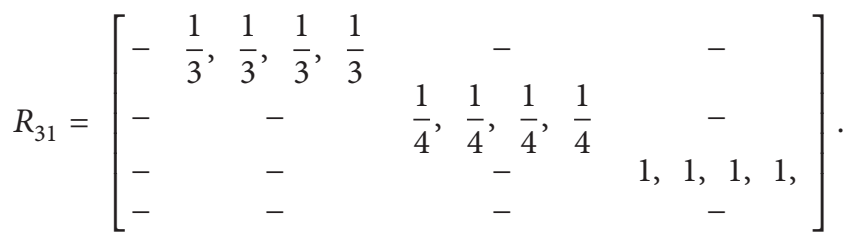

The preference relations' assessments of Attribute 2 which have been transformed into the type of trapezoidal membership function were given as follows:

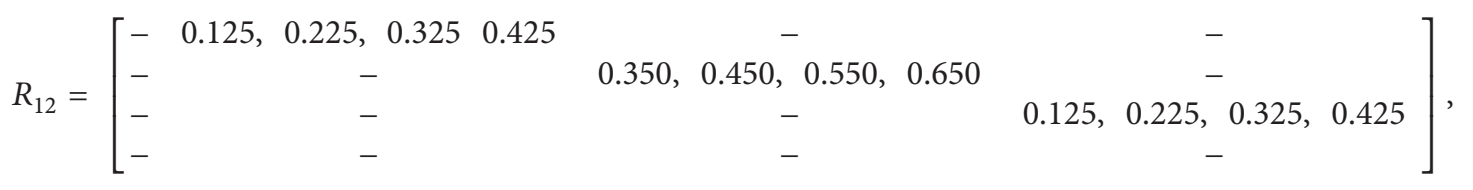

$$
\begin{aligned}
& R_{22}=\left[\begin{array}{ccccccc}
- & 0.050, & 0.150, & 0.250,0.350 & & - & - \\
- & - & 0.500,0.600,0.700,0.800 & - \\
- & - & - & - & 0.200,0.300,0.400,0.500 \\
- & - & & - & -
\end{array}\right] \text {, }
\end{aligned}
$$




$$
R_{32}=\left[\begin{array}{ccccccc}
- & \frac{1}{4}, & \frac{1}{4}, & \frac{1}{4}, & \frac{1}{4} & - & - \\
- & - & 1, & 1,1,1 & \\
- & - & - & \frac{1}{3}, & \frac{1}{3}, & \frac{1}{3}, & \frac{1}{3} \\
- & - & & - & & -
\end{array}\right]
$$

Using (1) the multiplicative preference relations in $R_{31}$ and $R_{32}$ can be transformed into fuzzy preference relations and

then become $R_{31}^{\prime}$ and $R_{32}^{\prime}$ as follows. $R_{31}$ and $R_{32}$ were then replaced by $R_{31}^{\prime}$ and $R_{32}^{\prime}$ for the rest of the analysis:

$$
\begin{aligned}
& R_{31}^{\prime}=\left[\begin{array}{ccccccc}
- & 0.250, & 0.250, & 0.250,0.250 & & - & - \\
- & - & - & 0.185, & 0.185, & 0.185,0.185 & - \\
- & - & - & - & 0.500,0.500,0.500,0.500 \\
- & & - & & - & -
\end{array}\right],
\end{aligned}
$$

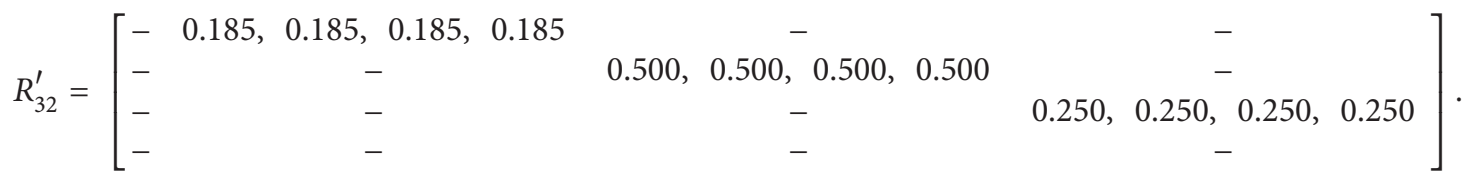

Step 2 (assessment aggregation for heterogeneous group of experts). In this example, the weights of Experts 1, 2, and 3 are $0.3,0.3$, and 0.4 , respectively. Following the method set out in Section 2.3, the six steps can be used to aggregate the assessments provided by the heterogeneous group of experts. Let the relaxation factor $\beta=0.5$. The results are then summarized in Table 1.

Therefore, the aggregated preference relations matrixes $\mathrm{PR}_{1}$ and $\mathrm{PR}_{2}$ are as shown in the following:

$$
\begin{aligned}
\mathrm{PR}_{1} & =\left[\begin{array}{cccc}
- & 0.290 & - & - \\
- & - & 0.311 & - \\
- & - & - & 0.500 \\
- & - & - & -
\end{array}\right], \\
\mathrm{PR}_{2} & =\left[\begin{array}{cccc}
- & 0.218 & - & - \\
- & - & 0.547 & - \\
- & - & - & 0.290 \\
- & - & - & -
\end{array}\right] .
\end{aligned}
$$

Step 3 (the generation of consistent preference relations). In Step 3, the results in $\mathrm{PR}_{1}$ and $\mathrm{PR}_{2}$ are incomplete. Equations (2) and (3) are then used to calculate the remaining preference relations and to construct additively consistent preference relation matrixes. The complete preference relation matrixes $\mathrm{PR}_{1}^{\prime}$ and $\mathrm{PR}_{2}^{\prime}$ are

$$
\begin{aligned}
\mathrm{PR}_{1}^{\prime} & =\left[\begin{array}{llll}
0.500 & 0.290 & 0.100 & 0.100 \\
0.710 & 0.500 & 0.311 & 0.311 \\
0.900 & 0.689 & 0.500 & 0.500 \\
0.900 & 0.689 & 0.500 & 0.500
\end{array}\right], \\
\mathrm{PR}_{2}^{\prime} & =\left[\begin{array}{llll}
0.500 & 0.218 & 0.265 & 0.055 \\
0.782 & 0.500 & 0.547 & 0.337 \\
0.735 & 0.453 & 0.500 & 0.290 \\
0.945 & 0.663 & 0.710 & 0.500
\end{array}\right] .
\end{aligned}
$$

According to the proposition and proof from HerreraViedma et al. [22], a fuzzy preference relation $\mathrm{PR}=\left(r_{i j}\right)$ is consistent if and only if $r_{i j}+r_{j k}+r_{k i}=3 / 2, \forall i \leq j \leq k$. It can be found that above $\mathrm{PR}_{1}^{\prime}$ and $\mathrm{PR}_{2}^{\prime}$ are consistent.

Step 4 (attribute weight determination). Using (15) to calculate all $\delta_{i k}$, the decision matrix DM can be constructed as follows:

$$
\mathrm{DM}=\left[\begin{array}{cc}
-2.019 & -1.923 \\
-0.336 & 0.331 \\
1.178 & -0.045 \\
1.178 & 1.637
\end{array}\right]
$$

According to the constructed decision matrix, when ADM and SDM were adopted, the weight of Attributes 1 and 2 can be calculated by (17) and (18), respectively. A value of $p=2$ has been adopted arbitrarily for the sake of this demonstration. If ADM is adopted, the weights of Attributes 1 and 2 are 0.501 and 0.499 , respectively. If SDM is adopted the weights of Attributes 1 and 2 are 0.509 and 0.491,respectively. 
TABLE 1: Aggregation of heterogeneous group of experts for Attribute 1.

\begin{tabular}{|c|c|c|c|}
\hline & $\widetilde{r}_{12}$ & $\widetilde{r}_{23}$ & $\widetilde{r}_{34}$ \\
\hline Expert 1 & $(0.125,0.225,0.325,0.425)$ & $(0.125,0.225,0.325,0.425)$ & $(0.350,0.450,0.550,0.650)$ \\
\hline Expert 2 & $(0.200,0.300,0.400,0.500)$ & $(0.350,0.450,0.550,0.650)$ & $(0.350,0.450,0.550,0.650)$ \\
\hline Expert 3 & $(0.250,0.250,0.250,0.250)$ & $(0.185,0.185,0.185,0.185)$ & $(0.500,0.500,0.500,0.500)$ \\
\hline \multicolumn{4}{|c|}{ Degree of agreement $\left(S_{a b}\right)$} \\
\hline$S_{12}$ & 0.925 & 0.775 & 1.000 \\
\hline$S_{13}$ & 0.900 & 0.880 & 0.900 \\
\hline$S_{23}$ & 0.875 & 0.685 & 0.900 \\
\hline \multicolumn{4}{|c|}{ Average degree of agreement of expert $a\left(\mathrm{AA}_{a}\right)$} \\
\hline $\mathrm{AA}_{1}$ & 0.913 & 0.828 & 0.950 \\
\hline $\mathrm{AA}_{2}$ & 0.900 & 0.730 & 0.950 \\
\hline $\mathrm{AA}_{3}$ & 0.888 & 0.783 & 0.900 \\
\hline \multicolumn{4}{|c|}{ Relative degree of agreement of expert $a\left(\mathrm{RA}_{a}\right)$} \\
\hline $\mathrm{RA}_{1}$ & 0.338 & 0.354 & 0.339 \\
\hline $\mathrm{RA}_{2}$ & 0.333 & 0.312 & 0.339 \\
\hline $\mathrm{RA}_{3}$ & 0.329 & 0.334 & 0.321 \\
\hline \multicolumn{4}{|c|}{$\begin{array}{l}\text { Consensus degree coefficient of expert } a\left(\mathrm{CC}_{a}\right) \text { for } \\
\beta=0.5\end{array}$} \\
\hline $\mathrm{CC}_{1}$ & 0.319 & 0.327 & 0.320 \\
\hline $\mathrm{CC}_{2}$ & 0.317 & 0.306 & 0.320 \\
\hline $\mathrm{CC}_{3}$ & 0.364 & 0.367 & 0.361 \\
\hline Aggregated results & $\widetilde{r}_{12}=(0.19,0.26,0.32,0.38)$ & $\widetilde{r}_{23}=(0.22,0.28,0.34,0.41)$ & $\widetilde{r}_{34}=(0.40,0.47,0.53,0.60)$ \\
\hline Converted results & $r_{12}=0.290$ & $r_{23}=0.311$ & $r_{34}=0.500$ \\
\hline
\end{tabular}

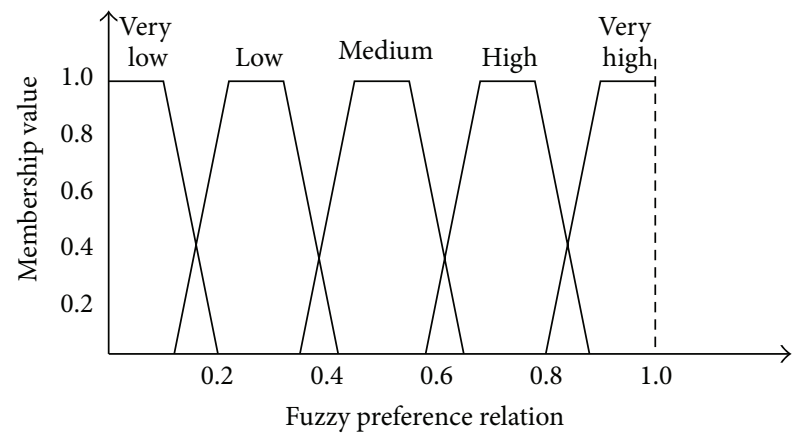

Figure 2: Membership functions adopted by Expert 1.

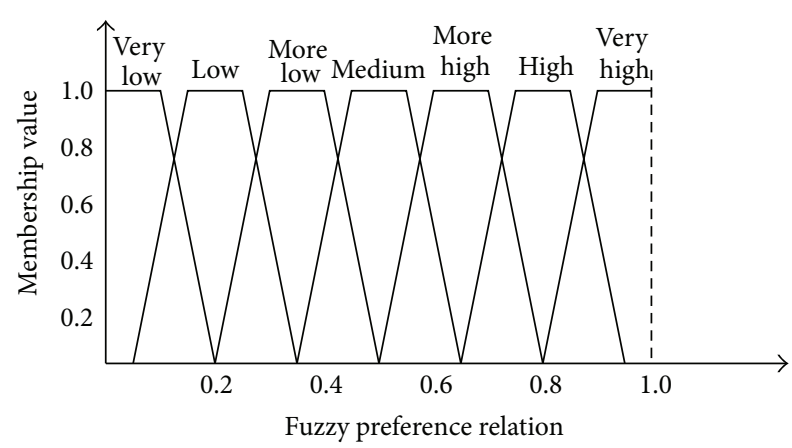

FIgURE 3: Membership functions adopted by Expert 2.
TABLE 2: The scoring results by weight determination methods ADM and SDM.

\begin{tabular}{lccccc}
\hline Alternative $i$ & \multicolumn{2}{c}{$\delta_{i k}$} & $C_{i}(\mathrm{ADM})$ & $C_{i}(\mathrm{SDM})$ & Ranking results \\
\hline 1 & -2.019 & -1.923 & -1.971 & -1.972 & 4 \\
2 & -0.336 & 0.331 & -0.003 & -0.009 & 3 \\
3 & 1.178 & -0.045 & 0.567 & 0.577 & 2 \\
4 & 1.178 & 1.637 & 1.407 & 1.403 & 1 \\
\hline $\mathrm{aw}_{k}$ by ADM & 0.501 & 0.499 & & & \\
$\mathrm{aw}_{k}$ by SDM & 0.509 & 0.491 & & & \\
\hline
\end{tabular}

Step 5 (ranking alternatives). After generating the weights of Attributes 1 and 2, using SAW, the score of all alternatives $C_{i}$ can be calculated by (9). The scoring results are as shown in Table 2. In Table 2, $C_{i}(\mathrm{ADM})$ and $C_{i}(\mathrm{SDM})$ indicate the scores of all alternatives using attribute weight determining approaches ADM and SDM, respectively. The bigger values of $C_{i}$ indicate that the alternative $i$ is better. In the case of the values of $C_{i}(\mathrm{ADM})$, for example, because $C_{4}(\mathrm{ADM})$ $>C_{3}(\mathrm{ADM})>C_{2}(\mathrm{ADM})>C_{1}(\mathrm{ADM})$, the group decision selected Alternative 4 as the first priority. Moreover, according to the values of $C_{i}(\mathrm{SDM})$, the results also show Alternative 4 as the first priority.

Although the theoretical development involves complicated technical details, the implementation is relatively straightforward in light of the numerical implementation. 
Therefore, the proposed methodology is applicable for a practical application. Its contribution can be justified accordingly.

\section{Conclusion}

This paper proposes a procedure for solving multiple attribute group decision making problems. In the proposed procedure, the transformation of assessment type, the property of consistency, the heterogeneity of a group of experts, the determination of weight, and scoring of alternatives are all considered. It would be a useful tool for decision makers in different industries. A review of the literature related to this research suggests that no previous research has addressed all of the issues simultaneously. The proposed procedure has several important properties as follows.

(i) Experts can provide their preference relations in various formats, which can then be transformed into a standard type.

(ii) Because all preference relation types are transformed into fuzzy preferences, and experts only provide preference relations between alternatives $i$ and $i+1$, it is possible to construct preference relations matrixes that satisfy the property of additive consistency.

(iii) Experts who are highly divergent from the group mean will have their weights reduced.

(iv) The weights of each attribute depend on the degree of variation; the higher the variation of the attribute, the higher its weight.

(v) Decision makers can select suitable MADM methods, such as SAW, GRA, or TOPSIS, for the final ranking step.

In the proposed procedure all the steps are adopted in response to observations made in the related literature and are understood by managers who are not experts in fuzzy theory, group decision making, MADM, or similar issues. A numerical example was described to illustrate the proposed procedure. It was demonstrated that the proposed procedure is simple and effective and can be easily applied to other similar practical problems.

The proposed procedure has some weaknesses in several of its properties. The weight of each expert depends on the divergence of his (or her) assessment from the opinions of other experts. Sometimes the real expert provides the most accurate assessment but is highly divergent from the mean of group. This characteristic would reduce the quality of the group decision. Moreover, the proposed procedure assumes that an attribute is quite important if the difference of the net degree of preference among all alternatives shows a wide variation. However, if an attribute is very important and has a relatively high weight, any small divergence in the assessment of the attribute can influence the ranking produced by the group decision. These weaknesses can provide the opportunity for future work.

\section{Conflict of Interests}

The authors declare that there is no conflict of interests regarding the publication of this paper.

\section{Acknowledgments}

This work was supported, in part, by the National Science Council of Taiwan under Grants NSC-101-2221-E-131-043 and NSC-101-2221-E-006-137-MY3.

\section{References}

[1] K. Yoon and C. L. Hwang, Multiple Attribute Decision Making: An Introduction, Sage, Thousand Oaks, Calif, USA, 1995.

[2] C. L. Hwang and K. Yoon, Multiple Attribute Decision Making: Methods and Applications, vol. 186 of Lecture Notes in Economics and Mathematical Systems, Springer, New York, NY, USA, 1981.

[3] T. L. Saaty, The Analytical Hierarchical Process, John Wiley \& Sons, New York, NY, USA, 1980

[4] A. Charnes, W. W. Cooper, and E. Rhodes, "Measuring the efficiency of decision making units," European Journal of Operational Research, vol. 2, no. 6, pp. 429-444, 1978.

[5] D. Ju-Long, "Control problems of grey systems," Systems \& Control Letters, vol. 1, no. 5, pp. 288-294, 1982.

[6] T. Yang and C. Kuo, "A hierarchical AHP/DEA methodology for the facilities layout design problem," European Journal of Operational Research, vol. 147, no. 1, pp. 128-136, 2003.

[7] T. Yang and C. C. Hung, "Multiple-attribute decision making methods for plant layout design problem," Robotics and Computer-Integrated Manufacturing, vol. 23, no. 1, pp. 126-137, 2007.

[8] T. Yang, Y.-C. Chang, and Y.-H. Yang, "Fuzzy multiple attribute decision-making method for a large 300-mm fab layout design," International Journal of Production Research, vol. 50, no. 1, pp. 119-132, 2012.

[9] T. Yang, Y.-F. Wen, and F.-F. Wang, "Evaluation of robustness of supply chain information-sharing strategies using a hybrid Taguchi and multiple criteria decision-making method," International Journal of Production Economics, vol. 134, no. 2, pp. 458-466, 2011.

[10] J.-C. Lu, T. Yang, and C.-T. Suc, "Analysing optimum push/pull junction point location using multiple criteria decision-making for multistage stochastic production system," International Journal of Production Research, vol. 50, no. 19, pp. 5523-5537, 2012.

[11] T. Yang and J.-C. Lu, "The use of a multiple attribute decisionmaking method and value stream mapping in solving the pacemaker location problem," International Journal of Production Research, vol. 49, no. 10, pp. 2793-2817, 2011.

[12] J. C. Lu, T. Yang, and C. Y. Wang, "A lean pull system design analysed by value stream mapping and multiple criteria decision-making method under demand uncertainty," International Journal of Computer Integrated Manufacturing, vol. 24, no. 3, pp. 211-228, 2011.

[13] J. Ma, J. Lu, and G. Zhang, "Decider: a fuzzy multi-criteria group decision support system," Knowledge-Based Systems, vol. 23, no. 1, pp. 23-31, 2010.

[14] F. J. Cabrerizo, I. J. Pérez, and E. Herrera-Viedma, "Managing the consensus in group decision making in an unbalanced fuzzy linguistic context with incomplete information," KnowledgeBased Systems, vol. 23, no. 2, pp. 169-181, 2010. 
[15] J. Guo, "Hybrid multicriteria group decision making method for information system project selection based on intuitionistic fuzzy theory," Mathematical Problems in Engineering, vol. 2013, Article ID 859537, 12 pages, 2013.

[16] F. Chiclana, F. Herrera, and E. Herrera-Viedma, "Integrating three representation models in fuzzy multipurpose decision making based on fuzzy preference relations," Fuzzy Sets and Systems, vol. 97, no. 1, pp. 33-48, 1998.

[17] F. Chiclana, F. Herrera, and E. Herrera-Viedma, "Integrating multiplicative preference relations in a multipurpose decisionmaking model based on fuzzy preference relations," Fuzzy Sets and Systems, vol. 122, no. 2, pp. 277-291, 2001.

[18] E. Herrera-Viedma, F. Herrera, and F. Chiclana, "A consensus model for multiperson decision making with different preference structures," IEEE Transactions on Systems, Man, and Cybernetics Part A:Systems and Humans., vol. 32, no. 3, pp. 394402, 2002.

[19] Z.-P. Fan, S.-H. Xiao, and G.-F. Hu, "An optimization method for integrating two kinds of preference information in group decision-making," Computers and Industrial Engineering, vol. 46, no. 2, pp. 329-335, 2004.

[20] Z.-P. Fan, J. Ma, Y.-P. Jiang, Y.-H. Sun, and L. Ma, "A goal programming approach to group decision making based on multiplicative preference relations and fuzzy preference relations," European Journal of Operational Research, vol. 174, no. 1, pp. 311-321, 2006.

[21] J. Zeng, M. An, and N. J. Smith, "Application of a fuzzy based decision making methodology to construction project risk assessment," International Journal of Project Management, vol. 25, no. 6, pp. 589-600, 2007.

[22] E. Herrera-Viedma, F. Herrera, F. Chiclana, and M. Luque, "Some issues on consistency of fuzzy preference relations," European Journal of Operational Research, vol. 154, no. 1, pp. 98109, 2004

[23] A. I. Ölçer and A. Y. Odabaşi, "A new fuzzy multiple attributive group decision making methodology and its application to propulsion/manoeuvring system selection problem," European Journal of Operational Research, vol. 166, no. 1, pp. 93-114, 2005.

[24] S. Bozóki, "Solution of the least squares method problem of pairwise comparison matrices," Central European Journal of Operations Research (CEJOR), vol. 16, no. 4, pp. 345-358, 2008.

[25] Y.-M. Wang and Z.-P. Fan, "Fuzzy preference relations: aggregation and weight determination," Computers \& Industrial Engineering, vol. 53, no. 1, pp. 163-172, 2007.

[26] F. E. Boran, S. Genç, M. Kurt, and D. Akay, "A multi-criteria intuitionistic fuzzy group decision making for supplier selection with TOPSIS method," Expert Systems with Applications, vol. 36, no. 8, pp. 11363-11368, 2009.

[27] F. J. Cabrerizo, S. Alonso, and E. Herrera-Viedma, "A consensus model for group decision making problems with unbalanced fuzzy linguistic information," International Journal of Information Technology and Decision Making, vol. 8, no. 1, pp. 109-131, 2009.

[28] S. J. Chuu, "Group decision-making model using fuzzy multiple attributes analysis for the evaluation of advanced manufacturing technology," Fuzzy Sets and Systems, vol. 160, no. 5, pp. 586602, 2009.

[29] J. Lu, Y. Zhu, X. Zeng, L. Koehl, J. Ma, and G. Zhang, "A linguistic multi-criteria group decision support system for fabric hand evaluation," Fuzzy Optimization and Decision Making, vol. 8, no. 4, pp. 395-413, 2009.
[30] Z. Zhang and X. Chu, "Fuzzy group decision-making for multiformat and multi-granularity linguistic judgments in quality function deployment," Expert Systems with Applications, vol. 36, no. 5, pp. 9150-9158, 2009.

[31] S. Cebi and C. Kahraman, "Developing a group decision support system based on fuzzy information axiom," KnowledgeBased Systems, vol. 23, no. 1, pp. 3-16, 2010.

[32] J. Kacprzyk, "Group decision making with a fuzzy linguistic majority," Fuzzy Sets and Systems, vol. 18, no. 2, pp. 105-118, 1986.

[33] J. Kacprzyk and M. Robubnes, Non-Conventional Preference Relations in Decision Making, Springer, Berlin, Germany, 1988.

[34] L. Kitainik, Fuzzy Decision Procedures with Binary Relations: Towards a Unified Theory, vol. 13, Kluwer Academic Publishers, Dordrecht, The Netherlands, 1993.

[35] T. Tanino, "Fuzzy preference orderings in group decision making," Fuzzy Sets and Systems, vol. 12, no. 2, pp. 117-131, 1984.

[36] S. A. Orlovsky, "Decision-making with a fuzzy preference relation," Fuzzy Sets and Systems, vol. 1, no. 3, pp. 155-167, 1978.

[37] H. M. Hsu and C. T. Chen, "Aggregation of fuzzy opinions under group decision-making," Fuzzy Sets and Systems, vol. 79, no. 3, pp. 279-285, 1996.

[38] S. M. Chen, "Aggregating fuzzy opinions in the group decisionmaking environment," Cybernetics and Systems, vol. 29, no. 4, pp. 363-376, 1998.

[39] J. Fodor and M. Roubens, Fuzzy Preference Modelling and Multicriteria Decision Support, Kluwer Academic Publishers, Dordrecht, The Netherlands, 1994.

[40] Y. Kuo, T. Yang, and G.-W. Huang, "The use of grey relational analysis in solving multiple attribute decision-making problems," Computers and Industrial Engineering, vol. 55, no. 1, pp. 80-93, 2008. 


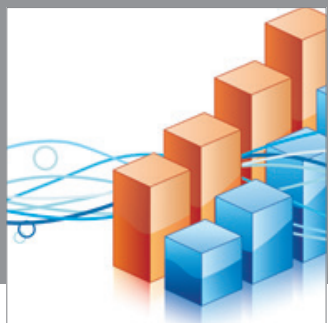

Advances in

Operations Research

mansans

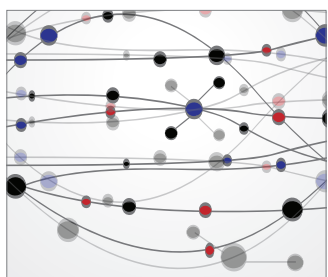

The Scientific World Journal
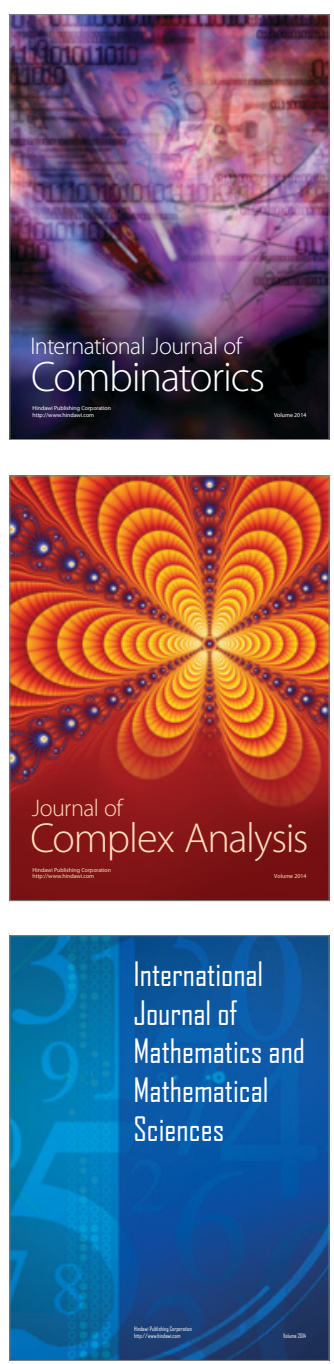
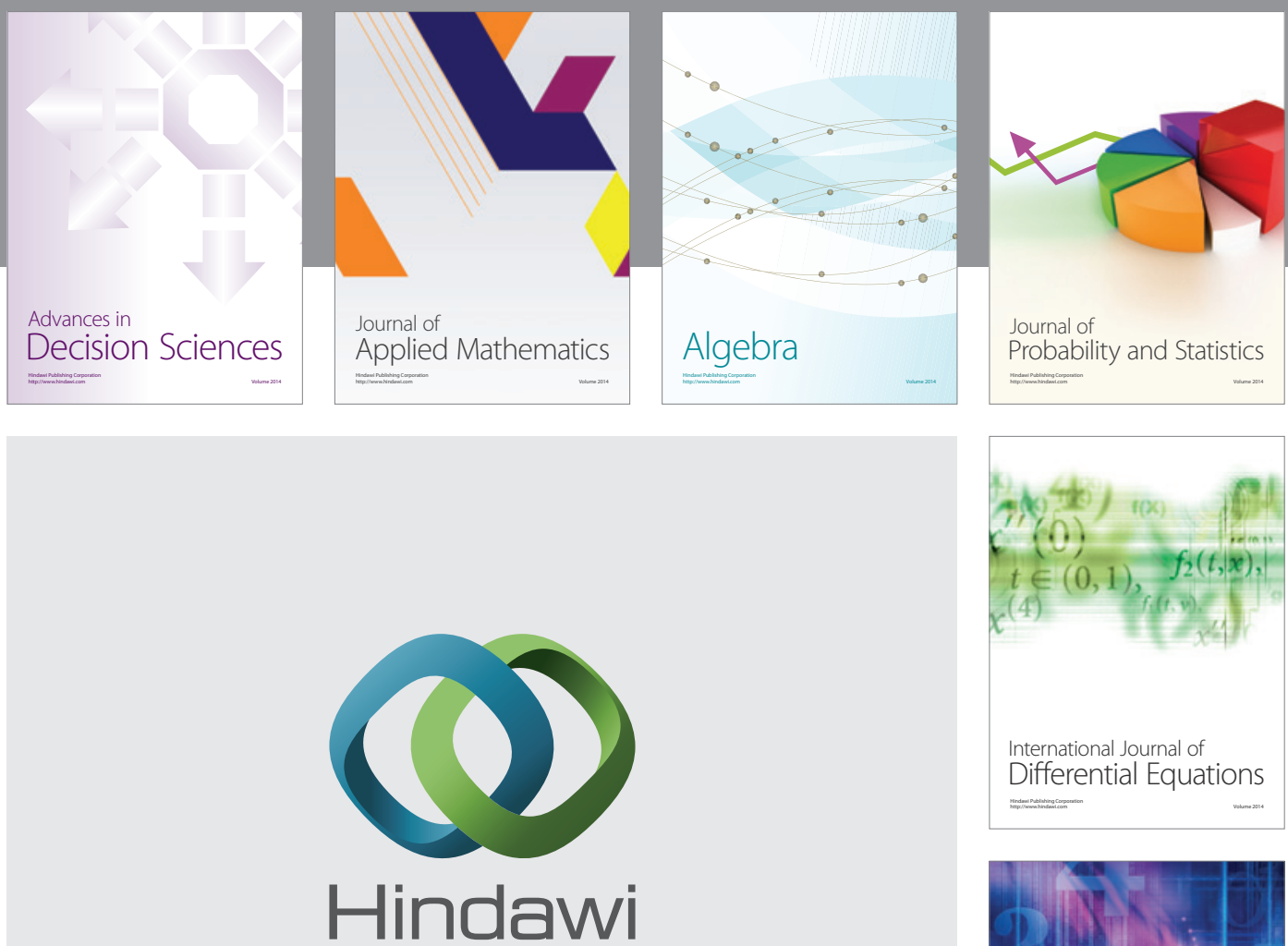

Submit your manuscripts at http://www.hindawi.com
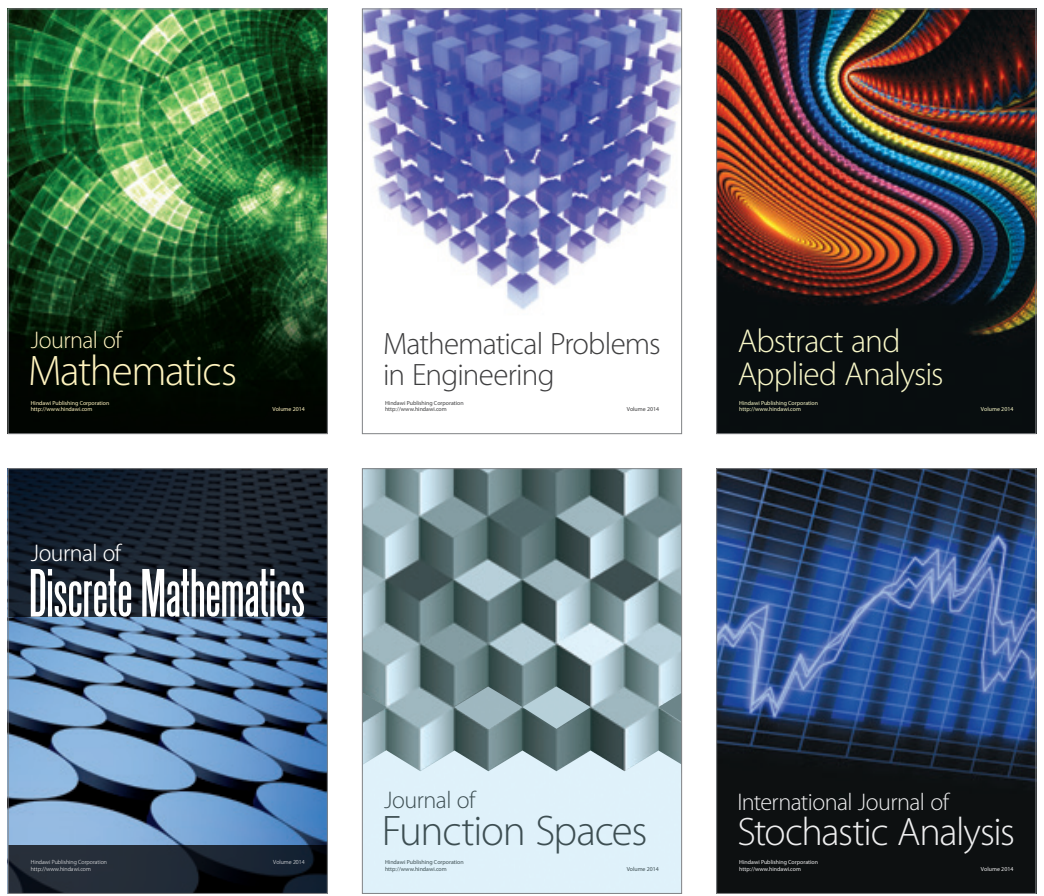

Journal of

Function Spaces

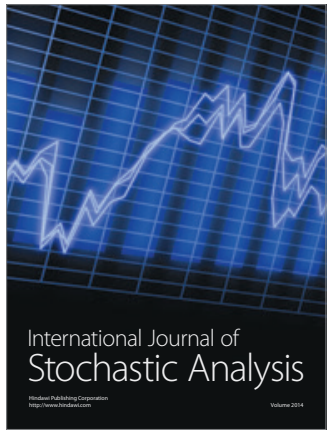

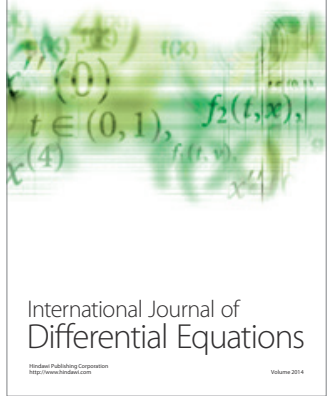
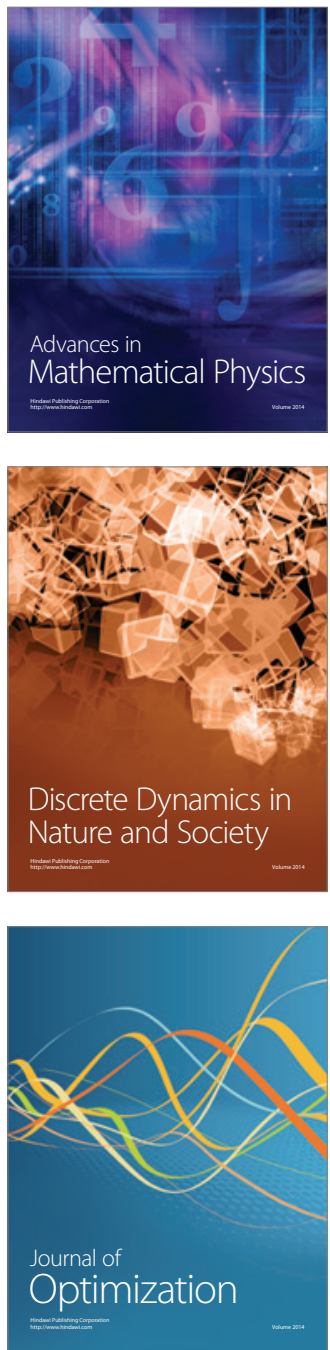